\title{
Update on future headache treatments
}

\author{
Abraham J. Nagy • Alan M. Rapoport
}

(C) Springer-Verlag Italia 2013

\begin{abstract}
Headache disorders are common and heterogenous neurologic entities. The complexities of management are further encumbered by the relatively few effective choices for acute and preventive therapies available to the headache specialist to treat these diverse disorders. As advances have been made in uncovering headache pathophysiology, new therapies have surfaced and others are forthcoming. This article will highlight new lines of care in development. There are several novel delivery mechanisms of familiar medications which bypass the limitations of current delivery systems, including the sumatriptan iontophoretic patch Zecuity, the intranasal sumatriptan OptiNose system, the zolmitriptan Rapidfilm orally dissolvable film and the orally inhaled dihydroergotamine Levadex system. New lines of care based upon recently discovered therapeutic targets will also be discussed including calcitonin gene-related peptide (CGRP) receptor antagonists, serotonin receptor agonists, and sphenopalatine ganglion (SPG) intermittent stimulation. Finally, emerging targets for future therapeutics will be explored including transient receptor potential vanilloid (TRPV1) receptor modulators,
\end{abstract}

A. J. Nagy $(\square) \cdot$ A. M. Rapoport

Nevada Headache Institute, 8205 W Warm Springs Rd,

Suite 210, Las Vegas, NV 89113, USA

e-mail: anagy@kecenters.com; nagyaj@nvhi.net

A. M. Rapoport

e-mail: alanrapoport@gmail.com

\section{A. J. Nagy}

K I E Centers for Advanced Medicine, LLC, Las Vegas, NV, USA

\section{A. M. Rapoport}

The International Headache Society, The David Geffen School of Medicine at UCLA, Los Angeles, CA, USA nitric oxide (NO) antagonists, gap junction modulators, glutamate receptor antagonists, orexin receptor antagonists and prostanoid receptor antagonists. Therapies developing over the next several years will be welcome additions to the headache specialist's armamentarium.

Keywords Acute migraine treatment - CGRP antagonist . Dihydroergotamine $\cdot$ Headache therapy pipeline

\section{Introduction}

Migraine is a common, episodic and chronic neurological disorder, involving the peripheral and central connections of the trigeminal nerve and the vasculature, and characterized by its severity and disability. Nearly $20 \%$ of the population of Western countries affected by migraine, probably migraine and chronic migraine, currently available therapeutic options seem to be remarkably limited. The most recent major advance in acute treatments available for patients occurred over two decades ago with the advent of triptans. Although onabotulinumtoxinA has been recently approved for chronic migraine, the progress with new alternatives for preventive medications seems to have slowed. Two beta blockers and two antiepileptics have remained the only FDA approved migraine preventives for many years. Cluster headache, considered as one of the most severe of the primary headache disorders, also has limited options for both prevention and acute care. Despite, the fact that few new therapeutic options have come to market, there has been a rapid evolution in the understanding of headache pathophysiology. Advances in imaging techniques, new animal models and innovative signaling molecules have opened the door to unique and exciting prospects for headache therapies. In this review, 
emerging treatments and new promising targets for drug development will be discussed.

\section{Innovations of older therapies}

\section{Triptan variations}

Injectable sumatriptan first was the first triptan launched in Europe in 1991 and the US in 1993. Today, there are seven triptans available in various formulations including tablets, injections, orally disintegrating tablets and nasal sprays. Although triptans currently represent the mainstay of acute migraine therapy [1], over one-third of the patients do not respond well to triptans and well over half of them are willing to try other treatments [2]. The main limitations of current triptans cited are slow speed of effect, recurrence of pain, poor tolerability, inadequate absorption of tablets and need for a second dose. Since sumatriptan has become generic, attempts at addressing these shortcomings have led to the design of several novel delivery mechanisms.

- Sumatriptan transdermal patch (Zecuity): One of the most novel approaches to delivering a medication, which may address the unmet need of acute treatment in the setting of nausea and vomiting or poor absorption due to migraine related gastric stasis, is the sumatriptan patch. NP101, is an iontophoretic transdermal patch that utilizes a small electric current to drive sumatriptan across the skin delivering a constant plasma level of $10 \mathrm{ng} / \mathrm{ml}$ throughout the $4 \mathrm{~h}$ dosing period more consistently than either the $100 \mathrm{mg}$ oral tablet or $20 \mathrm{mg}$ nasal preparation by bypassing the GI tract [3]. The microprocessor within the device continuously monitors skins resistance and adjusts drug delivery to maintain a consistent dose of $6.5 \mathrm{mg}$ of sumatriptan. The device has been demonstrated to be an effective acute care medication with few of the typical triptan associated side effects $[4,5]$. The $2 \mathrm{~h}$ pain relief was 53 vs. $29 \%$ placebo $(p=0.0001)$ and the $2 \mathrm{~h}$ pain free rate (primary endpoint) was 16 vs. $8 \%$ for placebo. The sustained pain relief rate from 2 to $24 \mathrm{~h}$ was $34 \%$ for the drug and $21 \%$ for placebo. The drug device also achieved significance over placebo with regard to nausea freedom, photophobia freedom and phonophobia freedom. The most common adverse event was application site-related pruritus, which was generally mild and resolved without treatment. With good $2 \mathrm{~h}$ pain relief, relief of associated symptoms, few adverse events and the ability to bypass the GI tract and first pass metabolism, this is an attractive alternative to standard oral treatment. NP101 was formerly called Zelrix but prior to launch has been rebranded in the US as Zecuity in association with its January 2013 approval by the FDA. It is expected to be available in the US in the fourth quarter of 2013.

- Intranasal sumatriptan via the Optinose intranasal apparatus: Current delivery systems for intranasal sumatriptan deliver suboptimal doses of sumatriptan because much of the dose tends to be swallowed and absorbed slowly via the GI tract. OptiNose, is a bi-directional, breath-actuated device that delivers sumatriptan powder more effectively than current technologies. Consisting of a mouthpiece and a nozzle which forms a seal when placed in one nostril, the device is activated by blowing into it. Upon actuation, the soft palate rises, isolating the nasal cavity from the oropharynx, and a fine powder of sumatriptan is deposited across the entire nasal mucosa on one side and then passes through to the other nostril. Because of the improved deposition of the drug, a phase I study was able to demonstrate that more sumatriptan is absorbed across the nasal cavity than traditional nasal delivery methods [6]. A phase II study was designed to evaluate the efficacy and safety of a single dose of 10 or $20 \mathrm{mg}$ of a powder formulation of sumatriptan delivered with the OptiNose device in comparison with placebo [7]. In terms of efficacy, a greater proportion of subjects who received sumatriptan 10/20 mg were painfree at $2 \mathrm{~h}$ compared with those who received placebo $(54 / 57 \%$ vs. $25 \%, p<0.05)$. Pain relief at $2 \mathrm{~h}$ for the two doses was significantly better than placebo (84/ $80 \%$ vs. $44 \%, p<0.001)$. Pain relief was noted as early as $60 \mathrm{~min}(73 / 74 \%$ vs. $38 \%, p<0.01)$ and at $48 \mathrm{~h}$, sustained pain-free results were also notable (47/ $49 \%$ vs. $27 \%, p<0.05)$. The most common adverse effect was dysgeusia or metallic taste in $10 \%$ of subjects receiving the $10 \mathrm{mg}$ dose and $13 \%$ receiving the $20 \mathrm{mg}$ dose. No significant adverse events or triptan sensations occurred, including chest discomfort, paresthesia or asthenia. The device is currently undergoing phase III investigations.

- Zolmitriptan orally dissolvable film: Orally dissolvable formulations of triptans appeal to migraine sufferers who have nausea and difficulty in ingesting conventional tablets. Zolmitriptan Rapidfilm advances this concept one step further and is a very thin polymeric film strip containing 2.5 and $5 \mathrm{mg}$ zolmitriptan. It averts the need for water and is design to be swallowed with saliva. Approved in early 2012 in Europe for acute migraine therapy, it is currently in the approval process by the FDA for North American distribution.

Orally inhaled dihydroergotamine (DHE): DHE is an ergot that has been available in various formulations for over the last 50 years. While intravenous DHE is the mainstay of headache 
inpatient management, it is limited in use for outpatient care. DHE administered via currently available nasal preparations has a slow onset of action, low bioavailability of about $40 \%$, and is less effective than nasal and subcutaneous sumatriptan [8]. Intramuscular and subcutaneous dihydroergotamine is more effective, but produces somewhat more adverse effects and the mode of administration is less convenient. A new oral DHE inhalation device, Levadex, has been demonstrated to provide similar onset of action and efficacy comparable to intravenous DHE. The device delivers DHE deep into the lung automatically after breath actuation. A phase I study of DHE delivered by the inhaler vs. $1 \mathrm{mg}$ of IV DHE was performed and revealed there was a rapid systemic absorption of DHE with a time to maximum concentration $\left(t_{\max }\right)$ of 12 min with a $0.88 \mathrm{mg}$ respirable dose (vs. a $6 \mathrm{~min} t_{\max }$ with the IV preparation) [9]. The ratio of AUC $0-\infty$ of inhaled vs. IV was approximately 0.77 , revealing slightly lower systemic levels of inhaled DHE than IV DHE.

Phase II data found that $32 \%$ of patients receiving $0.5 \mathrm{mg}$ doses achieved pain relief as early as $10 \mathrm{~min}$ ( $p=0.019)$ with a low incidence of side effects including nausea and no clinically significant change in pulmonary function tests, clinical lab findings, heart rate, blood pressure or respiratory rate [10]. Phase III trials demonstrated quick onset (pain relief was achieved in $30 \mathrm{~min}$ ) and very good efficacy, with acute pain relief (58.7\% of Levadex patients vs. $34.5 \%$ for placebo, $p=0.0001$ ) with low incidence of side effects, including nausea (4.5 vs. $2 \%$ ) and vomiting (2 vs. $0.1 \%$ ) [11]. This study also demonstrated a sustained pain freedom with a very low $6.5 \%$ recurrence rate at $24 \mathrm{~h}$ and $10.3 \%$ recurrence rate at $48 \mathrm{~h}$. This is notable especially when compared to two metaanalyses that have calculated the cumulative $24 \mathrm{~h}$ recurrence rate for triptans in general to between 22 and $29 \%$.

Additionally, Levadex was equally effective in treating patients early or late in the attack. Conclusions drawn from this data indicate that Levadex may be a good drug selection for patients not responding to oral triptans, those with severe nausea and vomiting, and as well as those who treat late into a migraine attack. Because of the long duration of action of DHE, this medication may be advantageous for menstrual migraine. At the time of this writing, Levadex is awaiting FDA approval.

\section{New agents and modalities}

Calcitonin gene-related peptide (CGRP) receptor antagonists

In the 1980s, CGRP was initially implicated in migraine pathophysiology based on it being a potent vasodilator; however, it is now understood to also be involved with sensory neurotransmission of meningeal trigeminovascular afferents as well as brain stem pain signaling [12]. CGRP receptors are compelling targets for acute migraine therapy and CGRP receptor antagonists may be the next most promising advance in migraine management. The first effective CGRP receptor antagonist was intravenous BIBN4096, olcegepant. Although it was reported in early clinical studies to produce headache relief in $66 \%$ of subjects compared to $27 \%$ of controls, the further development of the drug was terminated possibly because of low oral bioavailability and/or other unknown factors [13]. MK-0974, telcagepant, is the first reported oral CGRP antagonist to complete 2 phase III trials. It was found to be equally effective as zolmitriptan for acute migraine management with fewer side effects [14, 15], but liver function abnormalities arose when the drug was studied as a migraine preventive agent and further development was terminated [16]. A second Merck CGRP drug was studied and abandoned. Two other CGRP antagonists, BI 44370 TA and BMS927711, have recently shown promise [17, 18]. CGRP antagonists do not carry the same cardiovascular risk profile as triptans [19]. If approved by the FDA, the "gepant" class of medications would be the first non-serotonergic, non-vasoconstricting, migraine-specific line of care. There are apparently three companies developing antibodies to either CGRP or its receptor, but little is know about effectiveness and safety in patients at this time.

\section{Serotonin receptor agonists}

Triptans are high-affinity agonists for 5-hydroxytrypamine $(5-\mathrm{HT})_{1 \mathrm{~B}}$ and $5-\mathrm{HT}_{1 \mathrm{D}}$ receptors. Activation of $5-\mathrm{HT}_{1 \mathrm{~B}}$ receptors in the meninges accounts for the vasoconstrictive activity of triptans and the 5-HT $1 \mathrm{D}$ receptors activate presynaptic autoreceptors [20]. Some triptans are also agonists at the $5-\mathrm{HT}_{1 \mathrm{~F}}$ receptor [21] which are also located prejunctionally on the peripheral and central ends of sensory trigeminal neurons and have no vasoconstrictor action [22]. Activation of $5-\mathrm{HT}_{1 \mathrm{~F}}$ receptors is thought to be hyperpolarize nerve terminals and inhibit transmission of trigeminal nocioceptive signals [23]. COL-144, Lasmiditan, is a selective $5-\mathrm{HT}_{1 \mathrm{~F}}$ receptor agonist with 500 -fold less affinity at $5-\mathrm{HT}_{1 \mathrm{~B} / 1 \mathrm{D}}$ than 5-HT ${ }_{1 \mathrm{~F}}$ receptors [24]. Phase II studies demonstrated superiority over placebo for headache freedom at $2 \mathrm{~h}$; however, there was a dose-dependent central nervous system adverse effect profile consisting of dizziness, fatigue, vertigo, and somnolence [25]. Phase III studies are under development, and if supportive, the "ditan" class of medications may be another non-vasoconstrictive option for acute migraine therapy.

Sphenopalatine ganglion (SPG) stimulation

The field of neurostimulation for headache management is rapidly advancing. In various stages of development, 
modalities range from occipital nerve stimulation, supraorbital nerve stimulation, vagal nerve stimulation, deep brain stimulation of the posterior hypothalamus and transcranial magnetic stimulation. SPG stimulation is emerging as a viable therapeutic option for one of the most disabling disorders, chronic cluster headache. The SPG is located in the pterygopalatine fossa and contains parasympathetic and sympathetic fibers directly and indirectly connecting somatic and visceral nerve structures of the face to the trigeminovascular system, the superior salivatory nucleus in the brainstem and the hypothalamus. In cluster headache patients, low-frequency stimulation of the SPG can induce cluster-like attacks which can be aborted by high-frequency stimulation [26]. One proof of concept study found that in 6 patients, 11 out of 18 spontaneous or induced $\mathrm{CH}$ attacks were aborted by stimulating the SPG [27]. The Autonomic Technologies, Inc. (ATI) Neurostimulation System is a new wireless, rechargeable system using a remotely controlled implanted neurostimulator smaller than an almond. The device is designed to allow patients to deliver as-needed stimulation to relieve a cluster attack.

A recent multicenter, sham-controlled trial in Europe found that 19 of 28 patients had clinically significant benefit in acute pain relief [28]. Pain relief at 15 min was achieved in $67.1 \%$ of treated attacks compared to $7.4 \%$ of sham treated attacks $(p<0.0001)$, while pain freedom at 15 min was achieved in $34.1 \%$ of treated attacks compared to $1.5 \%$ of sham treated attacks $(p<0.0001)$. Interestingly the device may help to reduce the frequency of cluster attacks. With repeated stimulation, the average number of cluster attacks per week was reduced by $31 \%$ $(p=0.005)$, and $43 \%$ of patients experienced an average reduction of $88 \%$ in the number of attacks suffered. Adverse effects were similar to those reported in other orofacial, surgical procedures, with the most frequent being transient sensory disturbances in the territory of the maxillary nerve (V2). In a small trial for drug-resistant migraine, acute SPG stimulation was effective in 5 out 10 patients [29]. A larger trial for migraine is now being conducting in Europe.

\section{Emerging targets}

Transient receptor potential vanilloid (TRPV1) receptor modulators

TRPV1, also known as the capsaicin receptor and the vanilloid receptor 1 , is involved in the detection and regulation of body temperature as well as nociception. Located in both the central and peripheral trigeminal system, TRPV1 receptor activation leads to CGRP release [30].
- TRPV1 agonists: Civamide is a TRPV1 agonist and calcium channel blocker that selectively depletes type$\mathrm{C}$ nociceptive fiber release of excitatory neurotransmitters like CGRP and substance P. Civanex, which is $0.01 \%$ civamide nasal solution (Z-8-methyl- $N$-vanillyl-6-nonenamide, zucapsaicin), has shown promise for the management of cluster headache. In a meta-analysis of the two available studies, following 1 week of active treatment, there was a decrease in cluster pain of approximately 70 vs. $35 \%$ in the control group by the third week $(p=0.0312)$ [31]. Civanex was well tolerated and because it is not systemically absorbed, there were no systemic adverse effect or medication interactions reported. Subjects did have a burning sensation in the nose that began to dissipate over a few days and about the same time the cluster pain receded. Phase II and Phase III studies in episodic cluster headache have demonstrated the ability to significantly reduce cluster headache frequency [32].

One trial was conducted at 14 centers showing that $50 \mu \mathrm{g}$ of civamide was modestly effective in preventing cluster attacks. In another larger unpublished study of 112 subjects, civamide was effective at decreasing cluster attacks, but this was not was not statistically significant as the control solution contained saline which was suspected to have decreased cluster pain. A combined US and European phase III study is anticipated using only the vehicle as control [33]. If the results are positive, civamide may be an effective preventive for episodic cluster headache. Thus far, using various routes of administration including a patch, nasal spray, oral capsule, and topical cream, up to 1,600 patients have been treated with civamide in phase I to phase III studies for several indications. The only significant adverse effect has been a localized burning sensation at the site of application. Early studies in acute migraine reveal that civamide was only marginally effective [34].

- TRPVI antagonists: Unfortunately, there has been little supportive evidence for TRPV1 antagonists in chronic pain conditions; however, in principle it should be effective in migraine [35]. The results of a completed Phase II trial of the TRPVl antagonist SB-705498 for acute treatment of migraine, at the time of this writing, are not yet available [36].

Nitric oxide antagonism

Nitroglycerine, which is a pro-drug for nitric oxide (NO), has been known to be able to induce headache in normal volunteers [37] and migraine attacks in migraine suffers [38]. NO is involved with regulating vasculature diameter 
and is implicated in the pathogenesis of migraine by activating trigeminovascular fibers and causing release of CGRP [39, 40]. NO is synthesized from L-arginine by NO synthase. There are three isoforms of NO synthase: endothelial NOS (eNOS), neuronal NOS (nNOS) and inducible NOS (iNOS). $\mathrm{N}^{\mathrm{G}}$-L-Monomethyl arginine (L-NMMA) is a non-selective inhibitor of all three NOS enzymes [41] and lead to $-60 \%$ reduction in pain intensity during acute migraine attacks in a small trial [42]. Unfortunately, its clinical potential is rather limited due to its pharmacokinetic profile and vasoconstrictive propensity. iNOS-selective compounds had no effect in acute or preventive use [43], so current interest centers around other selective inhibitors for nNOS and eNOS. Thus far, pure nNOS or eNOS inhibitors have not reached clinical trials; however, $\mathrm{NXN}-188$, a molecule that combines $5-\mathrm{HT}_{1 \mathrm{~B} / 1 \mathrm{D}}$ receptor agonism with nNOS inhibition, is being actively researched [44]. Results of a phase II trial in acute migraine without aura failed to reach the primary endpoint (pain relief at $2 \mathrm{~h}$ ) but there was a statistically significant response for pain freedom, sustained pain freedom and use of rescue medication from 4 to $24 \mathrm{~h}$ [45]. In the clinically relevant endpoint of total migraine freedom (defined as complete absence of pain, nausea, photophobia and phonophobia) statistical significance was reached at $1 \mathrm{~h}(p=0.0284)$, and benefit was maintained throughout the study period. Although these results need to be confirmed, NXN-188 may have a role for patients who have attack recurrence (used in combination with a more rapidly acting drug) and possibly also as a preventive treatment.

\section{Gap junction modulators}

Cortical spreading depression (CSD), a wave of electrophysiological hyperactivity in the cortex followed by a wave of inhibition, has been attributed to being the pathophysiologic underpinning of migraine related aura and possibly even the underlying migraine process [46, 47]. Preventive medications, such as topiramate and valproate, suppress CSD in a dose-dependent manner [48]. CSD, in part, propagates from cell to cell via intercellular transmembrane conduits called gap junctions. SB-220453, tonabersat, is a gap-junction modulator and was effective in inhibiting CSD in animal models [49]. In addition, it reduces CSD-induced NO release and trigeminovascular responses. Unfortunately, two small randomized, controlled trials and a larger, well powered study were negative in terms of prevention of migraine without aura [5052]. One of these studies had an interesting design and recruited only patients who had migraine attacks with frequent aura. The primary endpoint of a reduction in migraine headache days with or without aura, was not met; however, a co-primary endpoint of this cross-over study was the reduction in the number of aura attacks. The investigators found a significant reduction of aura frequency by $71 \%$ in patients during tonabersat treatment when compared to the placebo arm of the trial. In two studies assessing its efficacy for acute migraine, Tonabersat was not superior to placebo; however, these trials were conducted with a hetergenous group of migraine patients (with and without aura) [53, 54]. Tonabersat may be a potential preventive option for migraine with aura, but not for migraine without aura.

\section{Glutamate receptor antagonists}

Glutamate is an excitatory neurotransmitter that activates two different receptors types, ionotropic glutamate receptors (iGluRs) and metabotropic glutamate receptors (mGluRs). iGluRs are ion channel pores directly activated by glutamate, whereas, mGluRs, when activated by glutamate, indirectly activate plasma membrane ion channels through guanine nucleotide-binding proteins (G-protein) signaling cascades. Glutamate and glutamate receptors have multiple sites of activity throughout the pathophysiological cascade of migraine and play a pivotal role in nociceptive trigeminovascular pain processing [55].

- iGluR antagonists: N-Methyl-D-aspartate (NMDA) glutamate receptors, one type of iGluR, are activated during CSD [56]. Ketamine, a potent NMDA receptor antagonist has demonstrated some effect in aborting migraine with aura attacks in 5 out of 11 patients with familial hemiplegic migraine [57]. Memantine, another NMDA receptor antagonist has been able to significantly decrease migraine frequency in two studies [58, 59]. In one, 36 out of 54 patients treated with memantine for at least 2 months reported a significant reduction in headache frequency. In the other, monthly headache frequency was reduced from 21.8 days at baseline to $16.1(p<0.01)$ at 3 months. The mean number of days with severe pain was reduced from 7.8 to 3.2 at 3 months $(p<0.01)$. The mean disability scores were also significantly reduced at 3 months when compared with baseline (36.6 vs 54.9, $p<0.01)$.

Two other iGluR types are $\alpha$-amino-3-hydroxy-5methyl-4-isoxazolepropionic acid (AMPA) receptors and kinate receptors. Tezampanel,LY293558, a mixed AMPA/kainate receptor antagonist has been demonstrated in early studies to be effective in the acute treatment of migraine and is well tolerated [60]. Phase III studies for acute migraine treatment are planned. Its oral pro-drug NGX426 is awaiting out-licensing for migraine and pain studies [61]. LY466195, a GLUK5 kainate receptor antagonist, is effective as a migraine treatment; however, its therapeutic potential may be 
limited because of mild reversible visual distortions [66]. Other compounds are in clinical development, including the AMPA receptor antagonist, BGG492 [62].

- mGluR antagonists. ADXI0059 is a mGluR5 negative allosteric modulator and may be an effective acute treatment for migraine. Early studies demonstrated $16.1 \%$ of patients were pain-free $2 \mathrm{~h}$ after oral ADXI0059 treatment compared to $4.5 \%$ after placebo $(p=0.039)$; however, there were a number of side effects such as dizziness, impaired concentration and visual disturbances (71\% compared to placebo $14 \%$ ) [63]. A large European multicenter, randomized, double-blind, placebo controlled, parallel-group, doseranging study is currently assessing ADX10059 for migraine prevention.

\section{Orexin receptor antagonists}

Integral to sleep and eating, Orexins are peptides that can alter the function of specific processes of the trigeminovascular system associated with migraine [64, 65]. Orexin $\mathrm{B}$, when injected into the posterior hypothalamus, can increase responses to dural stimulation in the A- and $\mathrm{C}$-fiber input neurons and resulted in increased spontaneous activity [66]. MK-6096, suvorexant, is a reversible orexin receptor antagonist to both orexin receptors, $\mathrm{OX}(1) \mathrm{R}$ and $\mathrm{OX}(2) \mathrm{R}$. It is currently in clinical development for insomnia [67], and a phase I study is in progress to evaluate it as a migraine preventive [68].

\section{Prostanoid receptor antagonists}

Nonsteroidal anti-inflammatory agents are common place in the armamentarium for acute headache management. Their mechanism of action is suspected to inhibit cyclooxygenase and decrease the synthesis of inflammatory mediators, like prostaglandin E2 (PGE2) which acts on EP2 and EP4 receptors. In the laboratory, it has been demonstrated that PGE2 can induce trigeminal mediated CGRP release [69]. During acute migraine attacks, elevated PGE2 levels have been measured in jugular venous blood [70]. Interestingly, intravenous PGE2 can induce migraine-like headaches in healthy subjects [71]. An EP4 receptor competitive antagonist, BGC20-1531, is currently undergoing a phase II evaluation for acute migraine.

\section{Conclusion}

As scientific advances have progressed over the last several decades, new distinct headache mechanisms have been uncovered and validated drug targets identified. The barriers to new therapeutic developments are being gradually surmounted. The next several years hold much promise for significant innovations in the management of headache disorders.

Parts of this paper were adapted from a previously published article, 1 year ago in this journal written by one of the authors [31].

Conflict of interest A. Nagy is on the Advisory Board of MAP. He is a speaker for Zogenix and Allergan. A. Rapoport is on the Advisory Boards of Nautilus Neurosciences, NuPathe, MAP and Winston. He is a speaker for Allergan, Impax, Nautilus Neurosciences and Zogenix and is an author of the Phase IIB study on telcagepant.

\section{References}

1. Johnston M, Rapoport A (2010) Triptans for the management of migraine. Drugs 70:1505-1518

2. Bigal M, Rapoport A, Aurora S et al (2007) Satisfaction with current migraine therapy: experience from 3 centers in US and Sweden. Headache 47:475-479

3. Pierce M, Marbury T, O'Neill C et al (2009) Zelrix: a novel transdermal formulation of sumatriptan. Headache 49:817-825

4. Goldstein J, Smith TR, Pugach N et al (2012) A sumatriptan iontophoretic transdermal system for the acute treatment of migraine. Headache 52:1402-1410. doi:10.1111/j.1526-4610. 2012.02198.x

5. Smith TR, Goldstein J, Singer R et al (2012) Twelve-month tolerability and efficacy study of NP101, the sumatriptan iontophoretic transdermal system. Headache 52:612-624. doi: 10.1111/j.1526-4610.2012.02094.x

6. Luthringer R, Djupesland PG, Sheldrake CD et al (2009) Rapid absorption of sumatriptan powder and effects on the glyceryl trinitrate model of headache following intranasal delivery using a novel bi-directional device. J Pharm Pharmacol 61:1219-1228

7. Djupesland PG, Dočekal P (2010) Intranasal sumatriptan powder delivered by a novel breath-actuated bi-directional device for the acute treatment of migraine: a randomised, placebo-controlled study. Cephalalgia 30:933-942

8. Boureau F, Kappos L, Schoenen J, Esperanca P, Ashford E (2010) A clinical comparison of sumatriptan nasal spray and dihydroergotamine nasal spray in the acute treatment of migraine. Int J Clin Pract 54:281-286

9. Armer T, Shrewsbury S, Newman S et al (2007) Aerosol delivery of ergotamine tartrate via a breath-synchronized plume-control inhaler in humans. Curr Med Res Opin 23:3177-3187

10. Shrewsbury S, Cook R, Taylor G et al (2008) Safety and pharmacokinetics of dihydroergotamine mesylate administered via a novel (TEMPO) inhaler. Headache 48:355-367

11. Aurora SK, Silberstein SD, Kori SH et al (2011) MAP0004, orally inhaled DHE: a randomized, controlled study in the acute treatment of migraine. Headache 51:507-517

12. Goadsby PJ, Edvinsson L (1993) The trigeminovascular system and migraine: studies characterizing cerebrovascular and neuropeptide changes seen in humans and cats. Ann Neurol 33:48-56

13. Olesen J, Diener HC, Husstedt IW et al (2004) Calcitonin gene related peptide receptor antagonist BIBN 4096 BS for the acute treatment of migraine. N Engl J Med 350:1104-1110

14. Ho TW, Mannix LK, Fan X et al (2008) Randomized controlled trial of an oral CGRP receptor antagonist, MK-0974, in acute treatment of migraine. Neurology 70:1304-1312 
15. Ho TW, Ferrari MD, Dodick DW et al (2008) Efficacy and to-lerability of MK-0974 (telcagepant), a new oral antagonist of calcitonin gene-related peptide receptor, compared with zolmitriptan for acute migraine: a randomised, placebo-controlled, parallel-treatment trial. Lancet 372:2089-2090

16. Tepper SJ, Cleves C (2009) Telcagepant, a calcitonin generelated peptide antagonist for the treatment of migraine. Curr Opin Investig Drugs 10:711-720

17. Diener HC, Barbanti P, Dahlöf C et al (2011) BI 44370TA, an oral CGRP antagonist for the treatment of acute migraine attacks: results from a Phase II study. Cephalalgia 31:573-584

18. Study to evaluate the PK of BMS-927711 in patients with migraine during acute migraine and non-migraine condition. NCT01445067 http://clinicaltrials.gov/ct2/show/NCT01445067 (accessed 2 Feb 2013)

19. Behm MO, Blanchard RL, Murphy MG, Chodakewitz JA, Palcza JS, Harris DE et al (2008) Assessment of the effect of MK-0974, an oral CGRP receptor antagonist, on spontaneous ischemia in patients with stable cardiovascular disease [abstract]. Headache 48:S39

20. Viallalon CM, Centurion D, Valdicia LF et al (2002) An introduction to migraine: from ancient treatment to functional pharmacology and antimigraine therapy. Proc West Pharmacol Soc 45:199-210

21. Neeb L, Meents J, Reuter U (2010) 5-HT1F receptor agonists: a new treatment option for migraine attacks? Neurotherapeutics 7:176-182

22. Färkkilä M, Diener H, Géraud G et al (2010) Lasmiditan (COL144), a selective 5HT1F agonist, is a rapid and effective oral treatment for acute migraine. J Headache Pain 11:S43

23. Shepheard S, Edvinsson L, Cumberbatch M, Williamson D, Mason G, Webb J et al (1999) Possible antimigraine mechanisms of action of the 5HT1F receptor agonist LY334370. Cephalalgia 19:851-858

24. Nelson DL, Phebus LA, Johnson KW (2010) Preclinical pharmacological profile of the selective 5-HTIF receptor agonist lasmiditan. Cephalalgia 30:1159-1169

25. Tfelt-Hansen PC, Olesen J (2012) The 5-HT1F receptor agonist lasmiditan as a potential treatment of migraine attacks: a review of two placebo-controlled phase II trials. J Headache Pain 13:271-275

26. Schytz H, Barløse M, Guo S, et al. (2013) Experimental activation of the sphenopalatine ganglion provokes cluster-like attacks in humans. Cephalalgia. doi: 10.1177/0333102413476370

27. Ansarinia M, Rezai A, Tepper S et al (2010) Electrical stimulation of sphenopalatine ganglion for acute treatment of cluster headaches. Headache 50:1164-1174

28. Schoenen J, Jensen R, Lanteri-Minet M, et al. (2013) Stimulation of the sphenopalatine ganglion (SPG) for cluster headache treatment. Pathway $\mathrm{CH}-1$ : a randomized, sham-controlled study. doi: 10.1177/0333102412473667

29. Tepper SJ, Rezai A, Narouze S et al (2009) Acute treatment of intractable migraine with sphenopalatine ganglion electrical stimulation. Headache 49:983-989

30. Jansen-Olesen I, Mortensen A, Edvinsson L (1996) Calcitonin gene-related peptide is released from capsaicin-sensitive nerve fibres and induces vasodilatation of human cerebral arteries concomitant with activation of adenylyl cyclase. Cephalalgia $16: 310-316$

31. Rapoport A (2012) The therapeutic future in headache. Neurol Sci 33:S119-S125

32. Saper J, Klapper J, Mathew N et al (2002) Intransal civamide for the treatment of episodic cluster headaches. Arch Neurol 59:990-994

33. Civamide nasal solution for cluster headache $(\mathrm{ECH})$. NCT 01341548. http://clinicaltrials.gov/ct2/show/NCT01341548 (accessed 2 Feb 2013)
34. Diamond S, Freitag F, Philips SB et al (2000) Intranasal civamide for the acute treatment of migraine headache. Cephalalgia 20:597-602

35. Voight EA, Kort ME (2010) Transient receptor potential vanilloid-1 antagonists: a survey of recent patent literature. Expert Opin Ther Pat 20:1107-1122

36. Use of SB-705498 in the acute treatment of migraine. NCT00269022. http://www.clinicaltrials.gov/ct2/show/NCT0026 9022 (accessed 2 Feb 2013)

37. Iversen HK, Olesen J, Tfelt-Hansen P (1989) Intravenous nitroglycerin as an experimental model of vascular headache. Basic characteristics. Pain 38:17-24

38. Thomsen L, Kruuse C, Iversen H, Olesen J (1994) A nitric oxide donor (nitroglycerin) triggers genuine migraine attacks. Eur Neurol 1:73-80

39. Olesen J, Thomsen LL, Iversen HK (1994) Nitric oxide is a key molecule in migraine and other vascular headaches. Trends Pharmacol Sci 15:149-153

40. Wei EP, Moskowitz MA, Boccalini P, Kontos HA (1992) Calcitonin gene-related peptide mediates nitroglycerin and sodium nitroprusside-induced vasodilation in feline cerebral arterioles. Circ Res 70:1313-1319

41. Griffith OW, Kilbourn RG (1996) Nitric oxide synthase inhibitors: amino acids. Methods Enzymol 268:375-392

42. Lassen LH, Ashina M, Christiansen I et al (1998) Nitric oxide synthase inhibition: a new principle in the treatment of migraine attacks. Cephalalgia 18:27-32

43. Høivik HO, Laurijssens BE, Harnisch LO et al (2010) Lack of efficacy of the selective iNOS inhibitor GW274150 in prophylaxis of migraine headache. Cephalalgia 30:1458-1467

44. Vaughan D, Speed J, Medve R, Andrews JS (2010) Safety and pharmacokinetics of NXN-188 after single and multiple doses in five phase I, randomized, doubleblind, parallel studies in healthy adult volunteers. Clin Ther 32:146-160

45. Medve RL, Lategan TW (2010) Assessment of the safety and tolerability of $\mathrm{NXN}-188$ dihydrochloride in healthy volunteers and migraineurs. J Headache Pain 11:S37

46. Hadjikhani N, Sanchez Del Rio M, Wu O et al (2001) Mechanisms of migraine aura revealed by functional MRI in human visual cortex. Prac Nat Acad Sci USA 98:4687-4692

47. Olesen J, Larsen B, Lauritzen M (1981) Focal hyperemia followed by spreading oligemia and impaired activation of $\mathrm{rCBF}$ in classic migraine. Ann Neurol 9:344-352

48. Ayata C, Jin H, Kudo C, Dalkara T, Moskowitz M (2006) Suppression of cortical spreading depression in migraine prophylaxis. Ann Neurol 59:652-661

49. Read SJ, Smith MI, Hunter AJ, Upton N, Parsons AA (2000) SB220453, a potential novel antimigraine agent, inhibits nitric oxide release following induction of cortical spreading depression in the anaesthetized cat. Cephalalgia 20:92-99

50. Hauge AW, Asghar MS, Schytz HW, Christensen K, Olesen J (2009) Effects of tonabersat on migraine with aura: a randomised, doubleblind, placebo-controlled crossover study. Lancet Neurol 8:718-723

51. Goadsby PJ, Ferrari MD, Csanyi A, Olesen J, Mills JG (2009) Randomized, double-blind, placebo-controlled, proof-of-concept study of the cortical spreading depression inhibiting agent tonabersat in migraine prophylaxis. Cephalalgia 29:742-750

52. Minster Pharmaceuticals. News \& media (2009) TEMPUS study results in migraine prevention. http://www.minsterpharma.com/ news_463.asp (accessed 8 Jan 2010)

53. Dahlöf C, Hauge A, Olesen J (2009) Efficacy and safety of tonabersat, a gapjunction modulator, in the acute treatment of migraine: a double-blind, parallelgroup, randomized study. Cephalalgia 29:S7-S16 
54. Silberstein S, Schoenen J, Gobel H et al (2009) Tonabersat, a gap-junctionmodulator: efficacy and safety in two randomized, placebo-controlled, dose-ranging studies of acute migraine. Cephalalgia 29:S17-S27

55. Johnson KW, Nisenbaum ES, Johnson MP et al (2008) Innovative drug development for headache disorders: glutamate. In: Olesen J, Ramadan NM (eds) Frontiers in Headache Research. United States, New York, pp 185-194

56. Gorji A, Scheller D, Straub H et al (2001) Spreading depression in human neocortical slices. Brain Res 906:74-83

57. Kaube H, Herzog J, Käufer T et al (2000) Aura in some patients with familial hemiplegic migraine can be stopped by intranasal ketamine. Neurology 55:139-141

58. Charles A, Flippen C (2007) Romero Reyes M, Brennan KC, Memantine for prevention of migraine: a retrospective study of 60 cases. J Headache Pain 8:248-250

59. Bigal M, Rapoport A, Sheftell F, Tepper D, Tepper S (2008) Memantine in the preventive treatment of refractory migraine. Headache 48:1337-1342

60. Sang CN, Ramadan NM, Wallihan RG et al (2004) LY293558, a novel AMPA/GluR5 antagonist, is efficacious and well tolerated in acute migraine. Cephalalgia 24:596-602

61. Raptor Pharmaceutical Corp. announces positive data on NGX426 in the potential treatment of neuropathic pain http://ir. raptorpharma.com/releasedetail.cfm?ReleaseID=633925 (accessed 2.Feb 2013)

62. Efficacy and safety of BGG492 in the treatment of migraine. NCT00892203. http://clinicaltrials.gov/ct2/show/NCT00892203 (accessed Feb 2, 2013)

63. Marin JC, Goadsby PJ (2010) Glutamatergic fine tuning with ADX-10059: a novel therapeutic approach for migraine? Expert Opin Investig Drugs 19:555-561
64. Bartsch T, Levy MJ, Knight YE, Goadsby PJ (2004) Differential modulation of nociceptive dural input to [hypocretin] orexin $\mathrm{A}$ and $\mathrm{B}$ receptor activation in the posterior hypothalamic area. Pain 109:367-378

65. Holland PR, Akerman S, Goadsby PJ (2006) Modulation of nociceptive dural input to the trigeminal nucleus caudalis via activation of the orexin 1 receptor in the rat. Eur $\mathrm{J}$ Neurosci 24:2825-2833

66. Holland P, Akerman S, Goadsby P (2006) Modulation of nociceptive dural input to the trigeminal nucleus caudalis via activation of the orexin 1 receptor in the rat. Eur $\mathrm{J}$ Neurosci 24:2825-2833

67. Winrow CJ, Gotter AL, Cox CD et al (2012) Pharmacological characterization of MK-6096 - a dual orexin receptor antagonist for insomnia. Neuropharmacology 62:978-987

68. A study of the safety and efficacy of MK-6096 for migraine prophylaxis in participants with episodic migraine (MK-6096020). NCT01513291. http://clinicaltrials.gov/ct2/show/NCT0151 3291 (accessed 2 Feb 2013)

69. Jenkins DW, Feniuk W, Humphrey PP (2001) Characterization of the prostanoid receptor types involved in mediating calcitonin gene-related peptide release from cultured rat trigeminal neurones. Br J Pharmacol 134:1296-1302

70. Sarchielli P, Alberti A, Codini M et al (2000) Nitric oxide metabolites, prostaglandins and trigeminal vasoactive peptides in internal jugular vein blood during spontaneous migraine attacks. Cephalalgia 20:907-918

71. Wienecke T, Olesen J, Oturai PS, Ashina M (2009) Prostaglandin E2 (PGE2) induces headache in healthy subjects. Cephalalgia 29:509-519 